\title{
Successful combination treatment of a bifocal secretory germinoma with brain stem compression in a 17-year-old girl
}

\author{
Justyna Chalubinska-Fendler ${ }^{1}$, Monika Bulas², Malgorzata Klonowicz ${ }^{1}$, Wojciech Fendler ${ }^{2}$, \\ Jacek Fijuth ${ }^{1}$, Wojciech Mlynarski²
}

\begin{abstract}
${ }^{1}$ Department of Radiotherapy, Medical University of Lodz, Lodz, Poland ${ }^{2}$ Department of Paediatrics, Oncology, Haematology and Diabetology, Medical University of Lodz, Lodz, Poland
\end{abstract}

Submitted: 8 August 2014

Accepted: 18 Septeber 2014

Arch Med Sci 2016; 12, 3: 678-680

DOI: 10.5114/aoms.2016.59941

Copyright (c) 2016 Termedia \& Banach

\author{
Corresponding author: \\ Justyna Chalubinska-Fendler \\ MD, PhD \\ Department of Radiotherapy \\ Medical University of Lodz \\ 4 Paderewskiego St \\ 93-509 Lodz, Poland \\ Phone: +48 426895567 \\ E-mail: justyna.chalubinska- \\ fendler@e.umed.lodz.pl
}

A 17-year-old girl, evaluated since 2007 for endocrinological disorders due to secondary amenorrhea and weight loss, was referred to the neurosurgery department in May 2011 due to symptoms of rapidly increasing intracranial pressure. Computed tomography revealed a bifocal, irregular, non-homogeneous tumour localised in pineal and suprasellar regions (Figures $1 \mathrm{~A}, \mathrm{~B}$ ). Immediate septostomy, ventriculo-peritoneal valve implantation and suprasellar tumour biopsy were performed. Histopathological examination confirmed the tumour to be a secretory type germinoma $(\beta-\mathrm{HCG}=0.696 \mathrm{mlU} / \mathrm{ml}$; AFP $=79.89 \mu \mathrm{g} / \mathrm{ml}$ in serum). After surgical biopsy (Figure $1 \mathrm{C}$ ) the chemotherapy was introduced according to the GCT SIOP96 protocol - which is the standard protocol for children in Poland with this diagnosis. The control magnetic resonance imaging (MRI) confirmed total regression of the suprasellar tumour and a residual pineal mass (Figure 1 D). At the end of July 2011 the patient was transferred to the Radiotherapy Department for treatment planning. Radiation therapy was initiated using both static and dynamic fields, including 3 target volumes (Figure $1 \mathrm{E}$ ): brain ventricles (blue) irradiated up to 30.6 Gy, PTV2 (cyan) - both tumour loci - up to 45.0 Gy with dose escalation on the pineal tumour residue up to 52.2 Gy (turquoise). Currently the patient is in 3.5-year follow-up (Figure $1 \mathrm{~F}$ ) in a good clinical condition, presenting only minor left-sided convergent strabismus, slight deafness for high frequency sounds and requires hormonal supplementation. The last MRI revealed only a residual mass in the pineal region $10 \times 7 \times 12 \mathrm{~mm}$ and a "flat" pituitary gland as deviations from the normal condition.

Intracranial germ cell tumours (GCTs) account for 1-2\% of all primary paediatric central nervous system (CNS) tumours and have a peak incidence at between 10 and 14 years with a male-female ratio of $2: 1$ [1]. Although multiple loci at presentation are associated with poorer prognosis [2], in this relatively unusual case, despite dramatic onset of symptoms, the treatment has proven successful thus far. Up to $20 \%$ of intracranial GCTs are multifocal, and they usually involve the suprasellar and pineal gland region. This is typically observed in adolescent males rather than females. Pineal GCTs induce acute symptoms of intracranial pressure which may be manifested by the classic triad of chronic symp- 

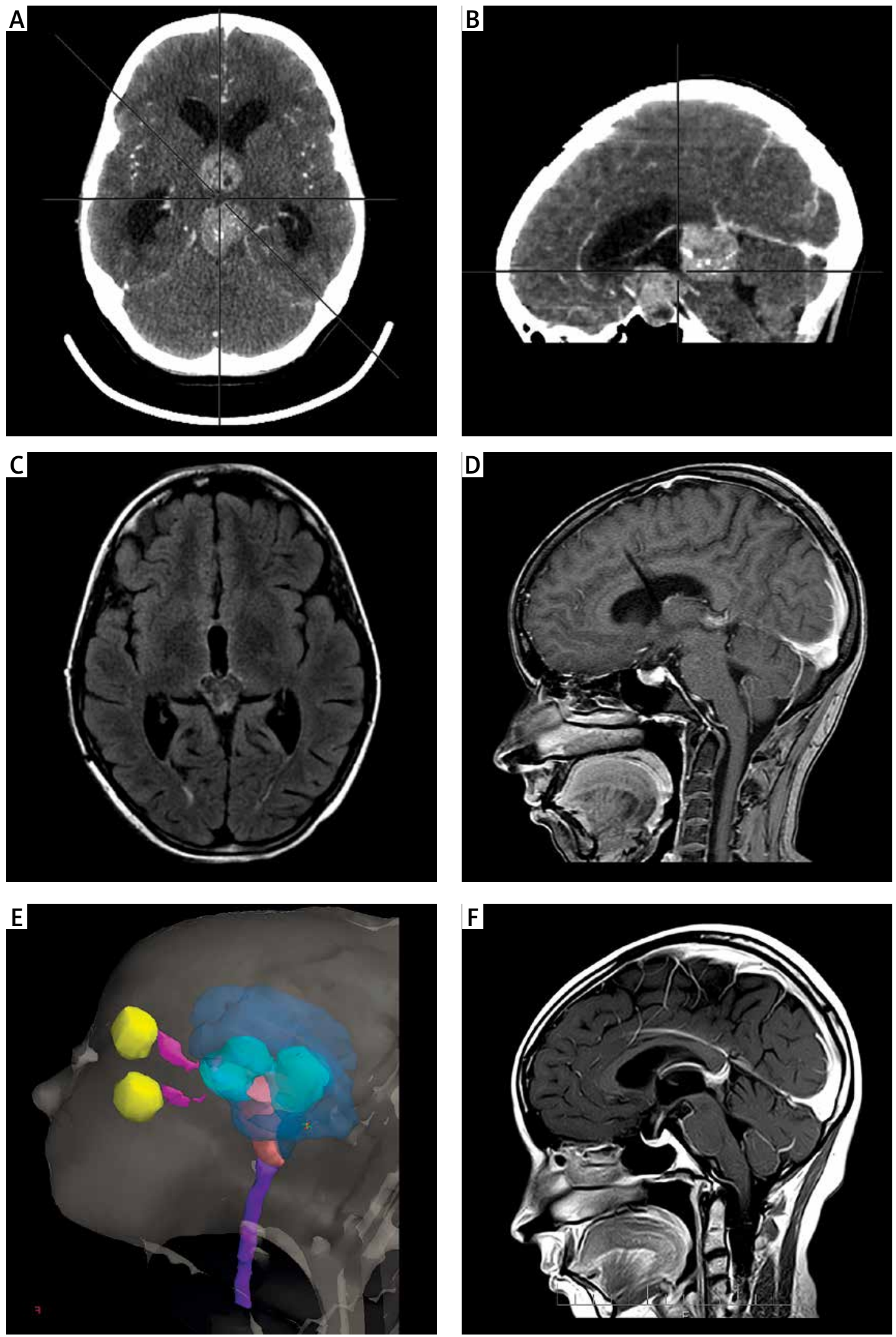

Figure 1. A, B - Transverse and sagittal computed tomography scans before initiation of treatment of pineal $(38 \times 30 \times 30 \mathrm{~mm})$ and suprasellar regions $(25 \times 20 \times 20 \mathrm{~mm}), \mathrm{C}-$ MRI scan after the surgical procedure, D - MRI showing pineal tumour reduction following chemotherapy $(16 \times 10 \times 16 \mathrm{~mm}), \mathrm{E}-3 \mathrm{D}$ planning before initiation of external beam radiation therapy; shades of blue represent critical regions of the tumour sites, F - CT at follow-up 2 years after initiation of treatment. No signs of the suprasellar mass are noted, with calcifications present within the site of the pineal mass 
toms: diabetes insipidus, visual deficits and precocious or delayed sexual maturity [3]. Evaluation must include MRI of the brain and serum marker levels (AFP, $\beta$-HCG) should be obtained. Biopsy sampling is recommended in all cases nowadays. Surgical treatment of intracranial GCTs remains controversial. Limited volume radiation therapy (whole ventricle followed by boost for residual tumour) is an important part of treatment preceded by chemotherapy according to currently used protocols. Endocrinopathies are a common complication of treating brain GCTs [4]. If pituitary gland insufficiency persists, it may however be easily corrected by hormonal treatment. The potential late sequelae due to chemotherapy and radiotherapy do not generally impair future mental and social development in adolescents, which gives a chance for a good quality of life in the future for these patients [5]. However, in younger patients, persistent neurological deficiencies resulting from surgical and radiotherapeutic complications may be a serious issue $[4,5]$.

\section{Acknowledgments}

Justyna Chalubinska-Fendler received financial support from the Lodz Region scholarship for outstanding PhD students. Wojciech Fendler and Wojciech Mlynarski received financial support from the TEAM programme of the Foundation for Polish Science financed by the European Union.

\section{Conflict of interest}

The authors declare no conflict.

\section{References}

1. Pizzo PA, Poplack DG. Principles and practice of pediatric oncology. $6^{\text {th }}$ ed. Lippincott Williams \& Wilkins, Philadelphia 2011.

2. Phi JH, Kim SK, Lee J, et al. The enigma of bifocal germ cell tumors in the suprasellar and pineal regions: syn chronous lesions or metastasis? J Neurosurg Pediatr 2013; 11: 107-14.

3. Halperin EC, Constine LS, Tarbell NJ, Kun LE. Paediatric radiation oncology. $5^{\text {th }}$ ed. Lippincott Williams \& Wilkins, Philadelphia 2010

4. Strojan P, Zadravec LZ, Anzic J, Korenjak R, Jereb B. The role of radiotherapy in the treatment of childhood intracranial germinoma: long-term survival and late effects. Pediatr Blood Cancer 2006; 47: 77-82.

5. Jinguji S, Yoshimura J, Nishiyama K, et al. Factors affecting functional outcomes in long-term survivors of intracranial germinomas: a 20-year experience in a single institution. J Neurosurg Pediatr 2013; 11: 454-63. 